\title{
House Sale Price Prediction using Advanced Regression Techniques and AutoML (TPOTRegressor)
}

\author{
V. S. B. Tejaswi ${ }^{1}$, K. V. V. Satyanarayana ${ }^{2}$ \\ ${ }^{1}$ Department of Computer Science and Engineering, Koneru Lakshmaiah Education Foundation,Vaddeswaram, Andhra \\ Pradesh, India, siriteja97@gmail.com \\ ${ }^{2}$ Department of Computer Science and Engineering, Koneru Lakshmaiah Education Foundation,Vaddeswaram, Andhra \\ Pradesh, India, kopparti@kluniversity.in
}

\begin{abstract}
The increasing and decreasing occurrence of house prices changes from time to time. There are more reasons that effect the fluctuation of house prices. Some are build year, location, physical amenities, size of house etc. Predicting the value of house (Sale price) helps the customers to take right choice of buying the house. Machine learning is being adapted for various fields that could build prediction model and estimate the outcomes. In this paper, we are contemplating the issue of rise and fall of house rates as a regression problem. Regression is a process that aims to predict the correlation between target dependent feature and a sequence of other changing independent features. In our experimental analysis, we are using Decision Tree Regression, Linear Regression, Ada Boost Regression, Gradient Boost Regression, Random Forest Regression techniques. In addition, we are also used AutoML to predict the House sale price. AutoML is a system that takes labelled trained data as input and automatically build a suitable optimized model that the dataset fits.
\end{abstract}

Key words: House price prediction, Machine learning Decision Tree Regression, Random Forest Regression, Gradient Boost Regression, Ada Boost Regression, AutoML.

\section{INTRODUCTION}

Day by day, the tendency of people liking to improve their living standards has been increasing. This is heading more of demand for houses. But, the problem is that customers may not know how much worth exactly a house to purchase. It may leads to take wrong decisions. Predicting the price or rate of a house is called house sale price prediction. It helps buyers and sellers. The price of the house varies now and then. The reason behind the changing value of house is based on many features. Some features are location, size of house, bedroom units, and count of storeys, living area, bathrooms, garage size, house age, type of roof and other utilities.

These are considered as independent features in which no feature has relation with other feature. The target feature to predict is Sale price. This is considered as dependent feature in which its value effects in changing the values of independent features.

Machine learning allows the machines to learn and to perform operations by themselves instead of inputting instructions explicitly. The machine learning project lifecycle workflows is as shown in below figure 1 .

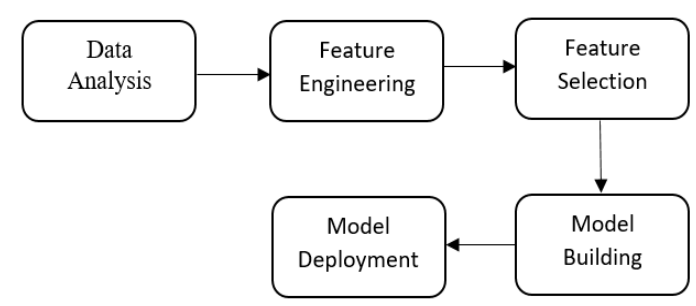

Figure 1: Machine learning project life-cycle

The first phase is Data Analysis. It is the process of visualizing data in means of graphs, finding missing values and observing correlations between features. Second phase is Feature Engineering. It is an activity of converting raw datasets into features which helps in improving the performance of machine learning techniques. Next one is feature selection. It is defined as a way of picking the most important features that effect more to predict the resultant outcome. Model building is defined as using machine learning techniques that enables model to learn from data without giving instructions. Model Deployment is the process of integrating built ML model to dynamic environment to make predictions from historical data.

Prediction in machine learning can be defined as generating an output from dataset input that is applied to a model. The model that best fits to a dataset implies an accurate prediction. We observed and implemented this problem using Regression analysis. Regression is a machine learning technique in which it can be performed whenever we need to model numerous independent features and to predict continuous dependent feature. Since predicting house price that is based on many independent changing features, we implemented regression analysis. We used Decision Tree Regressor, Random Forest Regressor, Linear Regressor, AdaBoost Regressor, Gradient Boost Regressor technique and AutoML. 
We organised this paper into five sections. First Section is Introduction. Second section demonstrates Data Preprocessing. Third section explains Algorithms, Forth section describes the model evaluation. Five and six sections are Conclusion and References.

\section{RELATED WORK}

Sifei $\mathrm{Lu}$ et al.[1] developedhybrid Lasso and Gradient boosting regression technique to predict the price of an individual house. Ayush Varma et al.[2] developed a system which predicts accurate house price and also used neural networks. Adyan Nur et al.[3] developed a swarn optimisation along with regression analysis to give optimum outcomes. G. Naga Satish et al.[4] developed a system which facilitates more functionalities to the vendor and used lasso regression. Nehal N Ghosalkar et al.[5] developed linear regression model and contemplated customer requirements and built according to it. D. Banerjee et al.[6] developed a system that utilised classification machine learning techniques. Bruno Klausde et al.[7] developed Recurrent neural networks, random forest and stated that this prediction helps house sellers and buyers. T. D. Phan et al.[8] developed SVM and neural networks and described that can be used for housing market. Sayan Putatunda et al.[9] developed random forests and gradient boosting which considers price as target outcome and all other features are independent features.

\section{DATA PRE-PROCESSING}

\subsection{House Price Dataset}

For experimental purpose, we imported House price datasets from https://www.kaggle.com. Dataset sample is as shown in below Figure 2 .

\begin{tabular}{|c|c|c|c|c|c|c|c|c|}
\hline Id & MSSubClass & MSZoning & LotFrontage & LotArea & Street & Alley & LotShape & LandContour \\
\hline 1 & 60 & RL & 65.0 & 8450 & Pave & $\mathrm{NaN}$ & Reg & Lvl \\
\hline 2 & 20 & RL & 80.0 & 9600 & Pave & $\mathrm{NaN}$ & Reg & LVI \\
\hline 3 & 60 & $\mathrm{RL}$ & 68.0 & 11250 & Pave & $\mathrm{NaN}$ & $\mid \mathrm{R} 1$ & Lvl \\
\hline 4 & 70 & RL & 60.0 & 9550 & Pave & $\mathrm{NaN}$ & |R1 & Lvl \\
\hline 5 & 60 & RL & 84.0 & 14260 & Pave & $\mathrm{NaN}$ & $\mid \mathrm{R} 1$ & Lvl \\
\hline
\end{tabular}

Figure 2: Dataset sample

\subsection{Dataset Description}

The description of dataset is representing in below Figure 3.

\begin{tabular}{|c|c|c|}
\hline Variable & Data type & Data description \\
\hline Id & numeric & Identity number \\
\hline MSSubClass & numeric & The building class \\
\hline MSZoning & text & The general zoning classification \\
\hline LotFrontage & numeric & Linear feet of street connected to property \\
\hline LotArea & numeric & Lot size in square feet \\
\hline Street & text & Type of road access \\
\hline Alley & text & Type of alley access \\
\hline LotShape & text & General shape of property \\
\hline LandContour & text & Flatness of the property \\
\hline Utilities & text & Type of utilities available \\
\hline LotConfig & text & Lot configuration \\
\hline LandSlope & text & Slope of property \\
\hline Neighborhood & text & Physical locations within Ames city limits \\
\hline Condition1 & text & Proximity to main road or railroad \\
\hline Condition 2 & text & Proximity to main road or railroad (if a second is present) \\
\hline BldgType & text & Type of dwelling \\
\hline HouseStyle & text & Style of dwelling \\
\hline OverallQual & numeric & Overall material and finish quality \\
\hline OverallCond & numeric & Overall condition rating \\
\hline YearBuilt & numeric & Original construction date \\
\hline YearRemodAdd & numeric & Remodel date \\
\hline RoofStyle & text & Type of roof \\
\hline RoofMatl & text & Roof material \\
\hline Exterior1st & text & Exterior covering on house \\
\hline Exterior2nd & text & Exterior covering on house (if more than one material) \\
\hline MasVnrType & text & Masonry veneer type \\
\hline MasVnrArea & numeric & Masonry veneer area in square feet \\
\hline ExterQual & text & Exterior material quality \\
\hline ExterCond & text & Present condition of the material on the exterior \\
\hline Foundation & text & Type of foundation \\
\hline BsmtQual & text & Height of the basement \\
\hline BsmtCond & text & General condition of the basement \\
\hline BsmtExposure & text & Walkout or garden level basement walls \\
\hline BsmtFinType1 & text & Quality of basement finished area \\
\hline BsmtFinSF1 & numeric & Type 1 finished square feet \\
\hline BsmtFinType2 & text & Quality of second finished area (if present) \\
\hline BsmtFinSF2 & numeric & Type 2 finished square feet \\
\hline BsmtUnfSF & numeric & Unfinished square feet of basement area \\
\hline TotalBsmtSF & numeric & Total square feet of basement area \\
\hline Heating & text & Type of heating \\
\hline Heating $Q \mathrm{CC}$ & text & Heating quality and condition \\
\hline CentralAir & text & Central air conditioning \\
\hline Electrical & text & Electrical system \\
\hline 1stFlrSF & numeric & First Floor square feet \\
\hline 2ndFlrSF & numeric & Second floor square feet \\
\hline LowQualFinSF & numeric & Low quality finished square feet (all floors) \\
\hline GrLivArea & numeric & Above grade (ground) living area square feet \\
\hline BsmtFullBath & numeric & Basement full bathrooms \\
\hline BsmtHalfBath & numeric & Basement half bathrooms \\
\hline FullBath & numeric & Full bathrooms above grade \\
\hline HalfBath & numeric & Half baths above grade \\
\hline Bedroom & numeric & Number of bedrooms above basement level \\
\hline Kitchen & numeric & Number of kitchens \\
\hline KitchenQual & text & Kitchen quality \\
\hline TotRmsAbvGrd & numeric & Total rooms above grade (does not include bathrooms) \\
\hline Functional & text & Home functionality rating \\
\hline Fireplaces & numeric & Number of fireplaces \\
\hline FireplaceQu & text & Fireplace quality \\
\hline GarageType & text & Garage location \\
\hline GarageYrBlt & numeric & Year garage was built \\
\hline GarageFinish & text & Interior finish of the garage \\
\hline GarageCars & numeric & Size of garage in car capacity \\
\hline GarageArea & numeric & Size of garage in square feet \\
\hline GarageQual & text & Garage quality \\
\hline GarageCond & text & Garage condition \\
\hline PavedDrive & text & Paved driveway \\
\hline WoodDeckSF & numeric & Wood deck area in square feet \\
\hline OpenPorchSF & numeric & Open porch area in square feet \\
\hline EnclosedPorch & numeric & Enclosed porch area in square feet \\
\hline 3SsnPorch & numeric & Three season porch area in square feet \\
\hline ScreenPorch & numeric & Screen porch area in square feet \\
\hline PoolArea & numeric & Pool area in square feet \\
\hline PoolQC & text & Pool quality \\
\hline Fence & text & Fence quality \\
\hline MiscFeature & text & Miscellaneous feature not covered in other categories \\
\hline MiscVal & numeric & \$Value of miscellaneous feature \\
\hline MoSold & numeric & Month Sold \\
\hline YrSold & numeric & Year Sold \\
\hline SaleType & text & Type of sale \\
\hline SaleCondition & text & Condition of sale \\
\hline SalePrice & numeric & The property's sale price in dollars. \\
\hline
\end{tabular}

\subsection{Missing values}

There may be missing values in the dataset. Missing values in data may interpret inaccurate predictions. Considering missing values, we need to find the relation between missing values and dependent target feature. The objective of finding this relationship is to determine how much an individual feature's missing value is effecting the dependent feature. 
These are some of the visualizations (bar graphs) that shows relationship between individual missing value features and SalesPrice in below figures $4-8$.

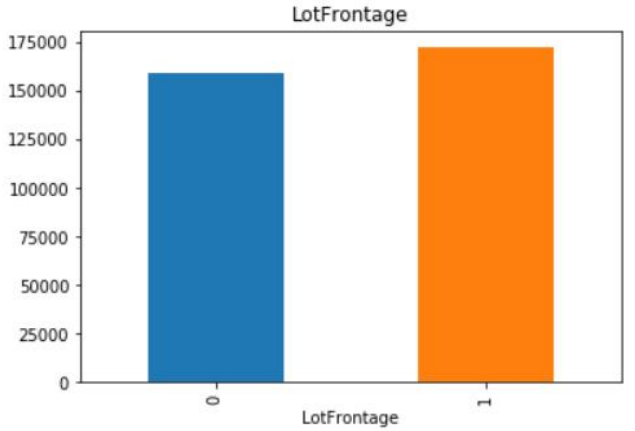

Figure 4: Relationship between LotFrontage's missing values and SalesPrice

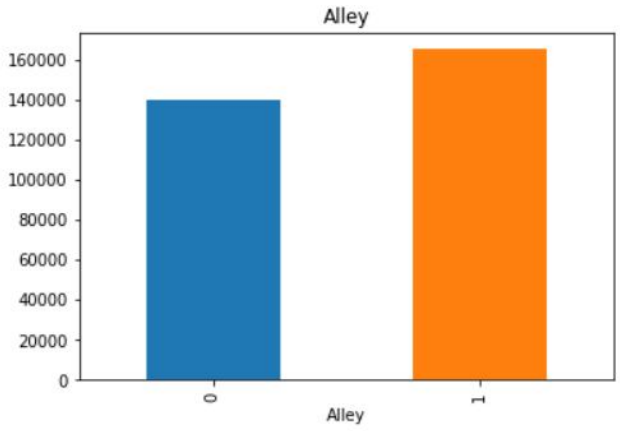

Figure 5:Relationship between Alley's missing values and SalesPrice

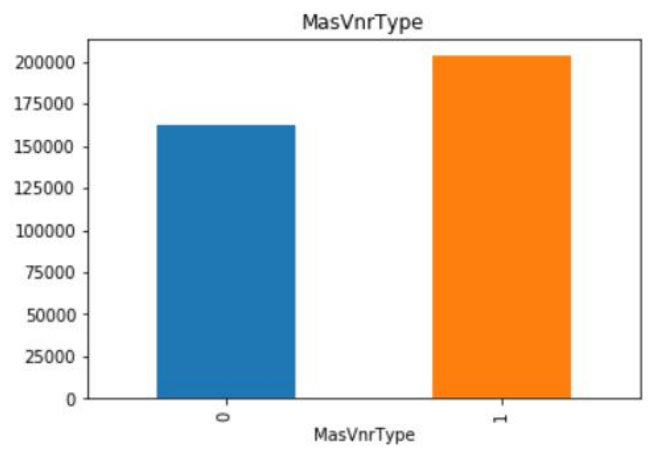

Figure 6:Relationship between MasVnrType's missing values and SalesPrice

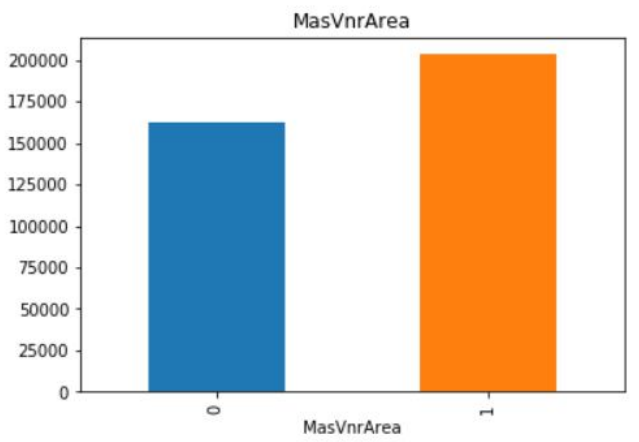

Figure 7: Relationship between MasVnrArea's missing values and SalesPrice

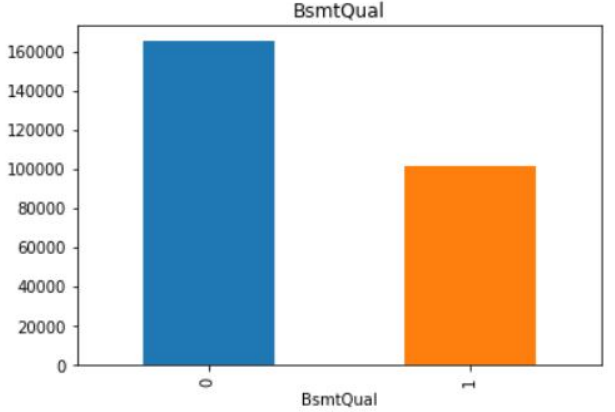

Figure 8:Relationship between BsmtQual's missing values and SalesPrice

In these graphs, ' 1 ' represents missing (Nan) values and ' 0 ' represents un-missing values. $\mathrm{X}$-axis represents independent feature and Y-axis represents SalesPrice. An efficient way to solve the issue of numeric missing values is to replace them with Mean/Median/Mode value. Another ways are replacing with zero/constant and repeated value within each column.

\subsection{Logarithmic transformation}

Logarithmic transformation is a technique which deals skewed data and reduce the data variability. The below scatter plot graphs figures $9-13$ presents logarithmic transformation of some continuous features.

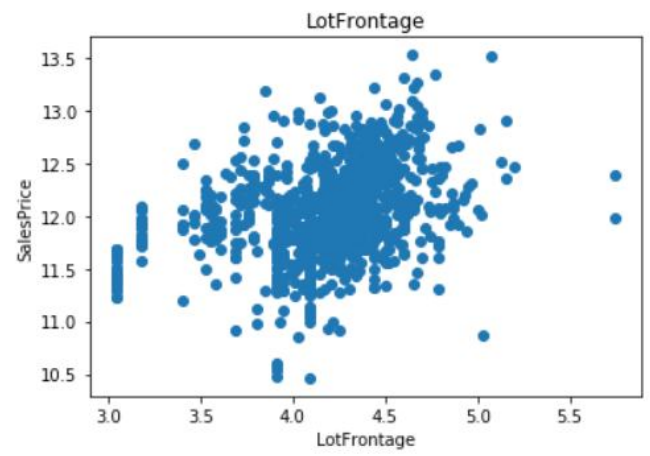

Figure 9: LotFrontage log transform

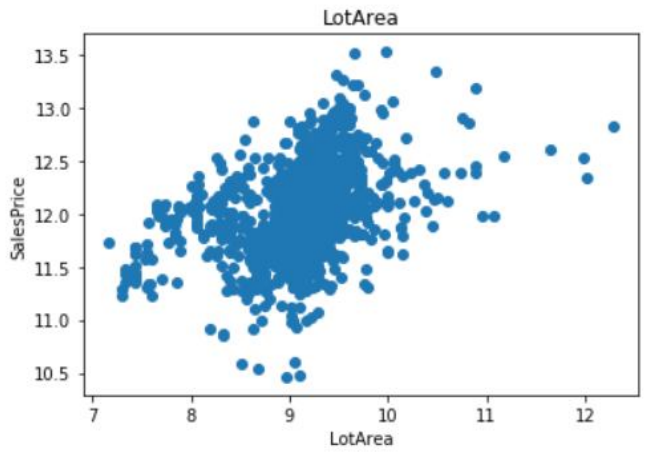

Figure 10: LotArea log transform 


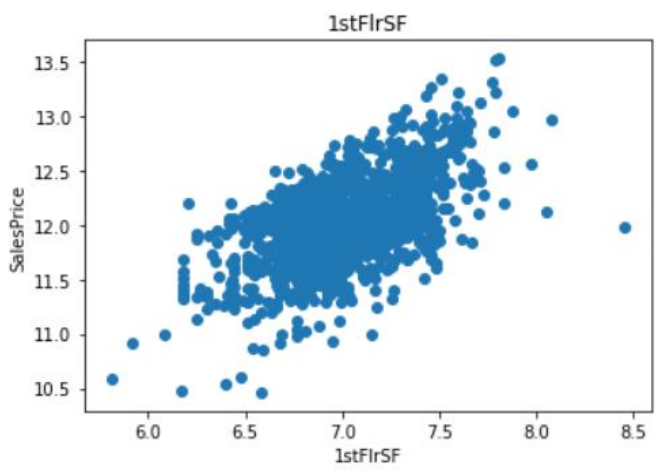

Figure 11:1stFlrSF log transform

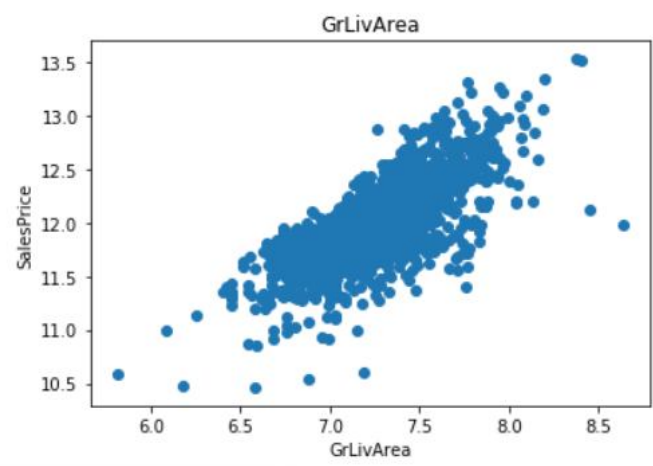

Figure 12: GrLivArea log transform

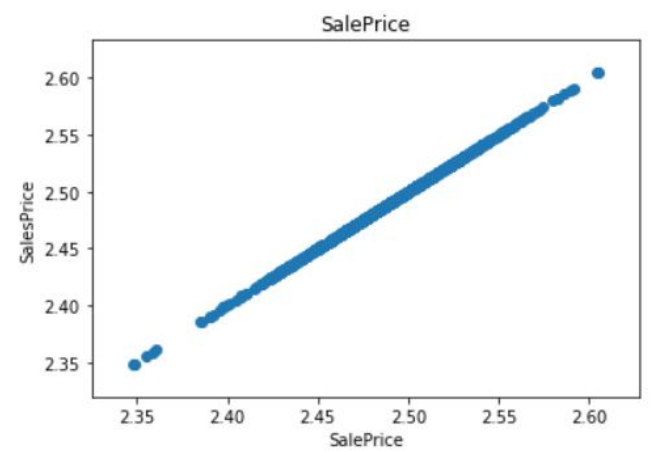

Figure 13: alePrice log transform

\subsection{Outliers}

Outliers are the extreme observations of data that deviates from rest of the data. The below boxplots figures $14-18$ are some of the observed outliers of continuous features.

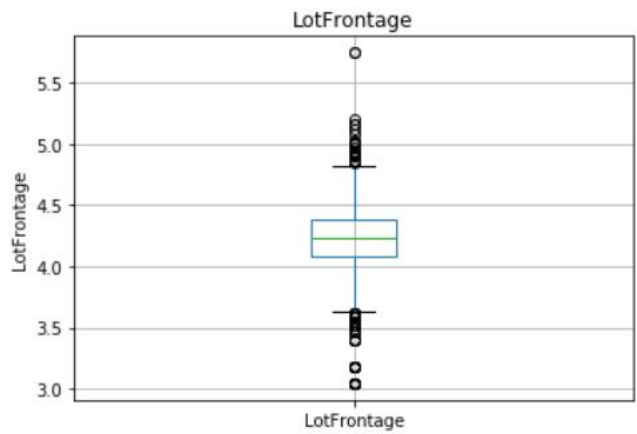

Figure 14: LotFrontage Outliers

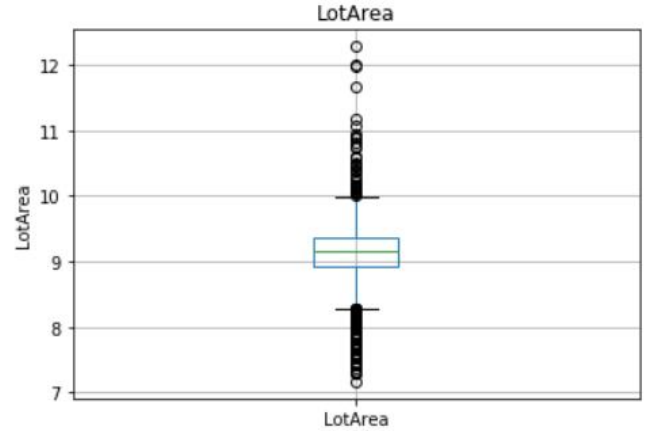

Figure 15: LotArea Outliers

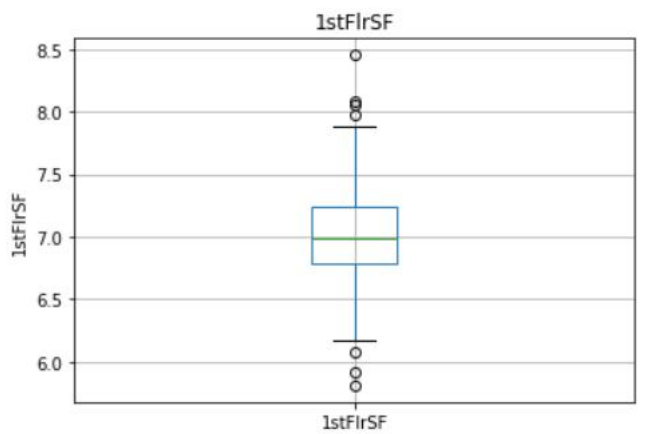

Figure 16:1stFlrSF Outliers

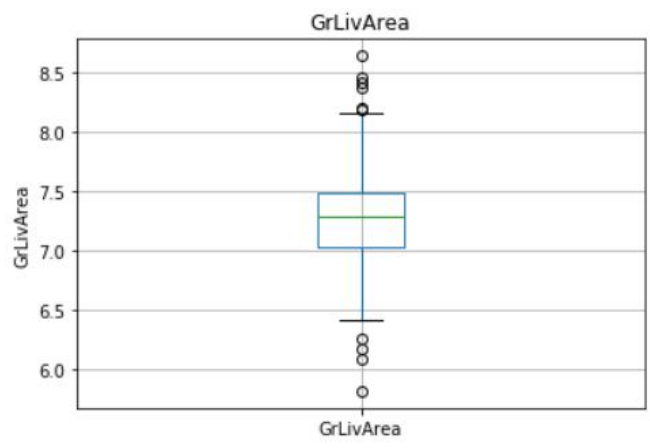

Figure 17: GrLivArea Outliers

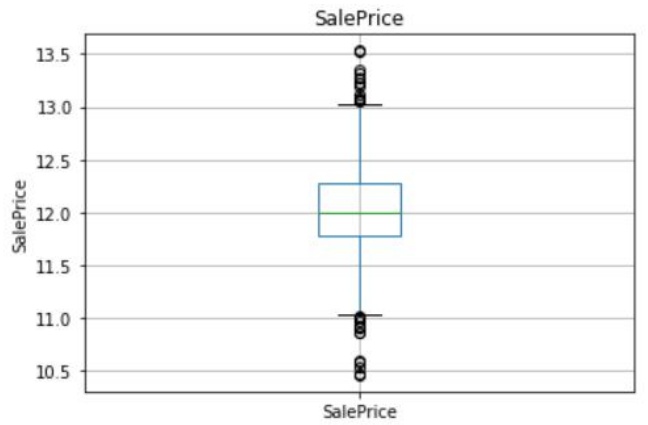

Figure 18: SalePrice Outliers

\subsection{Correlation}

Correlation is a quantify that shows how much a feature is dependent on another feature. The below heatmap figure 19 is showing how strongly one feature is related to other feature. 


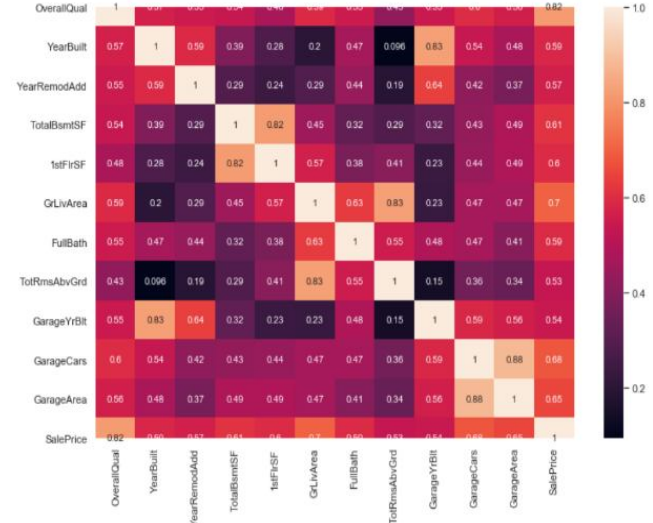

Figure 19: Correlation between features

\section{ALGORITHMS}

Before executing model, we consider the data into ' $\mathrm{x}$ ' and ' $y$ '. $x$ consists of input of independent features. $y$ consists of output of dependent feature. Here, y is SalePrice which is targeted feature. We split this data into training data and

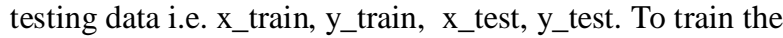
model, training data will be utilised. After we make the model to learn with train data, we give testing data (x_test) as input to the model. It estimates the outcome as y_test. The performance of a model can be measured in accuracy score. The accuracy score is difference between predict value score and real value score. $100 \%$ accuracy of a model leads to overfitting. Very least accuracy leads to underfitting.

\subsection{Linear Regression Technique}

Linear regression is one of the supervised machine learning methodology in which it performs on labelled data and predicts dependable feature from historical undependable features. It determines the linear relation of depend variable with independent variables.

\subsection{Random Forest Regression Technique}

Random forest regression is a supervised machine learning algorithm in which trees are executed parallel and generates mean prediction of each trees. Firstly it takes the sample input data and build number of decision trees. These decision trees run in parallel manner and estimate predictions. Considering all the predictions of trees, we calculate average for final result. Figure 20 shows random forest regression.

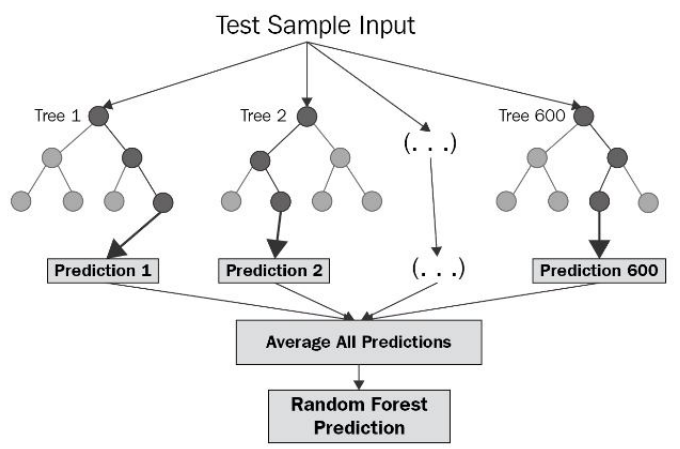

Figure 20: Random Forest regression workflow

\subsection{Gradient Boosting Regression}

Gradient boosting regression technique enables to construct a strong model upon weak regression model. Initially, it predicts using a model. Then it builds another model by solving the issues of first model. This process continues either whole dataset completes or until most of the model utilised. In our experimental analysis, it is proven that gradient boosting regressor predicted with high accuracy among other models.

\subsection{Ada Boost Regression}

Ada Boost Regression is a machine learning regression technique in which the predictions are estimated by taking mean of weak regressors. It is quick to detect the small changes of outliers and noisy data. Ada boost is alias name of Adaptive boosting.

\subsection{Decision Tree Regression}

Decision tree is one of the machine learning techniques in which dataset splits into small subsets and evaluate. Both continuous and categorical outcome features can be worked by decision tree. Decision tree regression considers some features as an input and trains the model to estimate the future outcome feature. Decision trees has an ability to observe the non-linear correlation of independent attributes with depend target attribute. Decision tree looks like following below figure 21 .

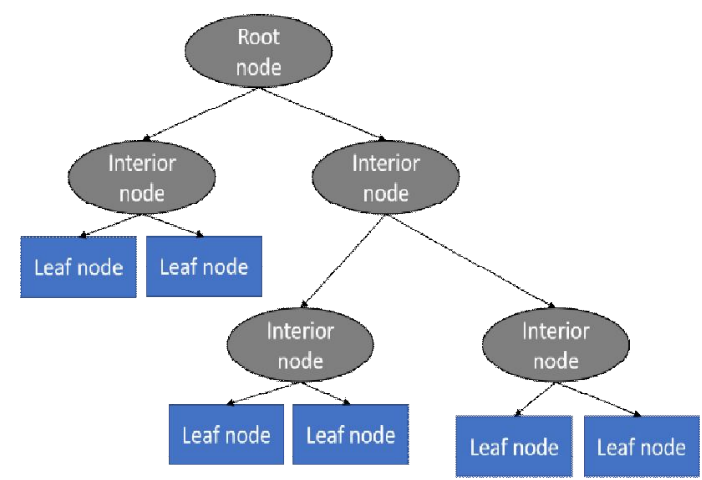

Figure 21:Decision Tree

\subsection{TPOT Regression (AutoML)}

Automated machine learning (AutoML) is a way of automatizing the computer to choose the model based on given dataset. So, it can give assistance to data scientists to pull the appropriate model. TPOT is an AutoML tool and the acronym is Tree-Based Optimization Tool. It provides open access to utilise packages. The procedure is same as other machine learning models. But the difference is, it automates the process of feature selection, feature preprocessing, feature construction, selection of model and parameter optimisation. In our experimental procedure, TPOT regressor chosen XGBRegressor as a model automatically based on given input dataset. Figure 22 shows TPOT regressor flow of work. 


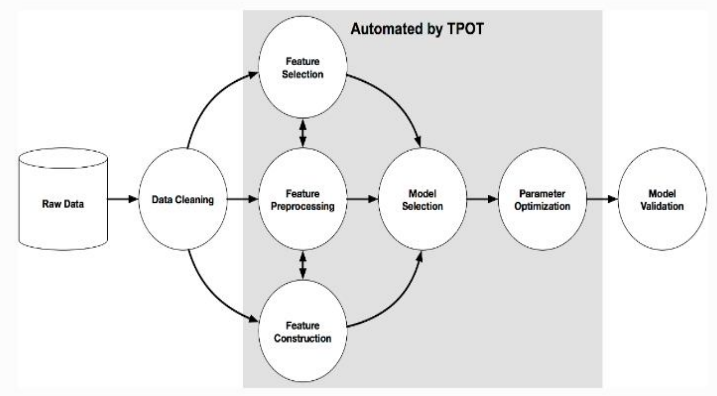

Figure 22:TPOT Regressor

\section{MODEL EVALUATION}

The accuracy score of all models are represented in the following below figure 23. Gradient boosting regressor model gave high accuracy among remaining models which is $91.8 \%$.

Random forest regressor and regressor got $89 \%$ accuracy. And the least with $76 \%$ accuracy score is Decision tree regression model. TPOT and Adaboost regressor estimated with the accuracy of $85 \%$.

\begin{tabular}{rrr} 
& Model & Score \\
\hline 2 & GradientBoostingRegressor & 91.823826 \\
1 & RandomForestRegressor & 89.442796 \\
0 & LinearRegression & 89.267087 \\
3 & AdaBoostRegressor & 85.055613 \\
5 & TPOT & 85.055613 \\
4 & DecisionTreeRegressor & 76.572470
\end{tabular}

Figure 23:Result Analysis

The accuracy score is also plotted in the below bar graph figure 24

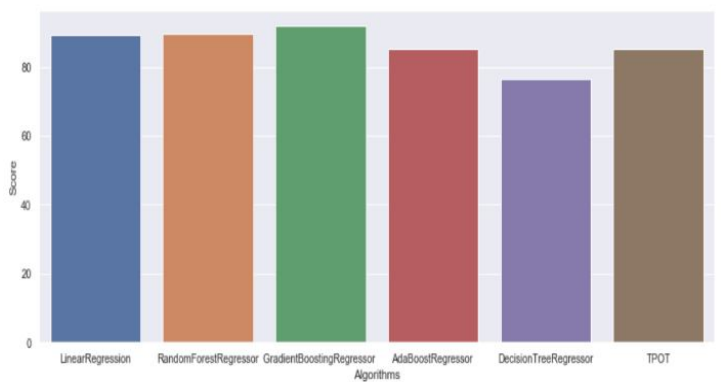

Figure 24:Results in bar graph

\section{CONCLUSION}

Prediction of house price is done using Regressor for house price dataset, comparing with different regression techniques like linear regression technique, decision tree regression algorithm, random forest regression technique, gradient boost regression, ada boost regression,. In further, TPOT regression (AutoML) is also performed. TPOT is an automatic tool that picks the best fit algorithm. The highest accuracy of $91 \%$ is from gradient boost regression. The customers and sellers will be benefitted with house price prediction.

\section{REFERENCES}

1. Sifei Lu, Zengxiang Li, Zheng Qin, Xulei Yang, Rick Siow Mong Goh, A Hybrid Regression Technique for House Prices Prediction, Institute of High Performance Computing (IHPC), Agency for Science Technology and Research (A*STAR), Singapore.

2. Ayush Varma, Abhijit Sarma, Sagar Doshi, Rohini Nair, House Price Prediction Using Machine Learning And Neural Networks.

3. Adyan Nur Alfiyatin, Hilman Taufiq, Ruth Ema Febrita, Wayan Firdaus Mahmudy, Modeling House Price Prediction using Regression Analysis and Particle Swarm Optimization.

4. G. Naga Satish, Ch. V. Raghavendran, M.D.Sugnana Rao, Ch.Srinivasulu, House Price Prediction Using Machine Learning.

5. Nehal N Ghosalkar, Sudhir N Dhage, Real Estate Value Prediction Using Linear Regression.

6. D. Banerjee and S. Dutta, Predicting the housing price direction using machine learning techniques, 2017 IEEE International Conference on Power, Control, Signals and Instrumentation Engineering (ICPCSI), Chennai, 2017, pp. 2998-3000. https://doi.org/10.1109/ICPCSI.2017.8392275

7. Bruno Klausde Aquino Afonso1, Luckeciano Carvalho Melo2, Willian Dihanster Gomesde Oliveira1, Samuel Brunoda Silva Sousa1, Lilian Berton1,Housing Prices Prediction with a Deep Learning and Random Forest Ensemble, Institute of Science and Technology - Federal University of S ao Paulo (UNIFESP)

8. T. D. Phan, Housing Price Prediction Using Machine Learning Algorithms: The Case of Melbourne City, Australia, 2018 International Conference on Machine Learning and Data Engineering (iCMLDE), Sydney, Australia, 2018, pp. $35-42$.

https://doi.org/10.1109/iCMLDE.2018.00017

9. Sayan Putatunda, PropTech for Proactive Pricing of Houses in Classified Advertisements in the Indian Real Estate Market 